Beobachtungen ron Cometen im Jahre 1877 auf der Sternwarte Kremsmilnster.

Comet b 1877.

Mai 10

12

17

27

28

Juni 2

3

$$
5
$$

8

10

11

12

14

15

16

17

18

19

20)

26

Juli 4

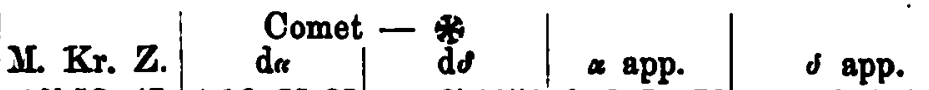
$10^{\mathrm{h}} \mathrm{2}^{\mathrm{m}} 47^{\mathrm{s}}+16^{\mathrm{m}} 25^{\circ} \mathrm{g} 5+6^{\prime} 18^{\prime \prime} 1^{\mathrm{h}} 16^{\mathrm{m}} \mathrm{g}^{\prime \prime} 73+77^{\circ} 23^{\prime} 34^{\prime \prime} 4$ $12.29 .46-54.02+1.36 .42 .37 .48 .72+79.37 .260$ $9.36 .19-1.53 .42-1.6 .36 .12 .9 .46+78.13 .32 .6$ $10.2438-43.96+57.18 .30 .4 .05+65.9 .47 .7$ $11.48 .30+4.3 .31-2.2 .88 .35 .38 .41+63.51 .30 .1$ $10.23 .36-5.17 .76+2.39 .08 .54 .9 .81+58.16 .52 .7$ 11.27. $8-9.34 .38-2.35 .18 .57 .9 .74+57.12 .37 .0$ $10.38 .15-6.8 .89+7.12 .58 .59 .4342+56.14 .59 .4$ $10.24 .46+51.75-10.46 .99 .2 .8 .86+5 \overline{0} .17 .58 .8$ 10.25. $7----$ - $-8.37 .40+52.34 .44 .1$ $10.13 .19-----0.12 .10 .12+$ ó 0.54 .49 .0 10. 5.39 - 9.39+2.58.7 9.14.0.75 + 50.7.8.6 10.14. $2-4.25 .85-4.35 .19 .15 .37 .20+49.20 .26 .4$ $958.58+1.41 .77-4.89 .18 .40 .43+47.51 .36 .3$ $10.14 .56-28.87-34.09 .19 .58 .31+47.9 .4 .5$ $10.19 .24+1.46 .00+10.45 .29 .21 .32 .44+46.28 .0 .3$ 10.17. $3-3.9 .91-2.42 .49 .22 .50 .10+4547.44 .5$ $10.31 .37---\mid--624.7 .93+4 \overline{0} .7 .59 .8$ $10.18 .35-7.44 .23-2.43 .29 .25 .22 .23+44.30 .17 .0$ 10.21.0 - - - - 9.26.36.13+43.53.9.9 $10.18 .14-1.14 .09+11.58 .39 .33 .10 .67+40.31 .15 .1$ $10.33 .45-4.9 .51-8.48 .19 .40 .45 .03+36.44 .5 .0$

\section{Comet c 1877.}

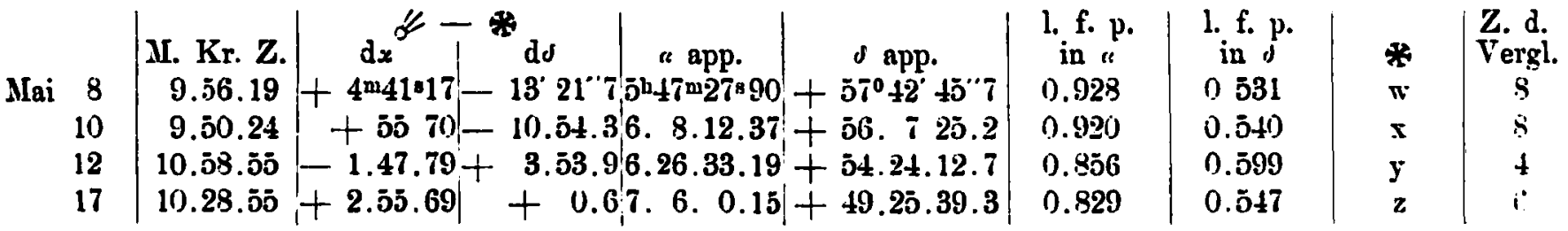

\begin{tabular}{|c|c|c|c|}
\hline $\begin{array}{l}\text { l. f. p. } \\
\text { in } a\end{array}$ & $\begin{array}{l}\text { 1. f. p. } \\
\text { in } \delta\end{array}$ & 3 & $\begin{array}{l}\text { Z. d. } \\
\text { Vergl. }\end{array}$ \\
\hline $0.799 n$ & 0.788 & $a$ & 2 \\
\hline $1.039 n$ & 0.754 & $\mathbf{b}$ & 12 \\
\hline 0.431 & 0.375 & c & 6 \\
\hline 1063 & 0.384 & d & 12 \\
\hline 0.994 & $0 . \overline{029}$ & $\mathbf{e}$ & 4 \\
\hline 0.920 & 0.359 & $f$ & 7 \\
\hline 0.873 & 0.494 & $\mathbf{g}$ & 4 \\
\hline 0.877 & 0.402 & $\mathbf{h}$ & 8 \\
\hline 0.702 & 0.377 & $\mathbf{i}$ & 6 \\
\hline 0.811 & 0.392 & $\mathbf{j}$ & 6 \\
\hline 0.782 & 0.376 & $\mathbf{k}$ & $S$ \\
\hline 0.769 & 0.365 & 1 & 10 \\
\hline 0.687 & 0.385 & $\mathbf{m}$ & 8 \\
\hline 0.729 & $0.35 \overline{6}$ & $\mathbf{n}$ & 8 \\
\hline 0.743 & 0.394 & 0 & 111 \\
\hline 0.706 & 0.402 & $\mathbf{p}$ & $\bar{\tau}$ \\
\hline $0.69 t$ & 0.401 & $q$ & 6 \\
\hline 0.667 & $0 . \pm 23$ & $\mathbf{r}$ & 8 \\
\hline 0.663 & 0.405 & $\mathbf{s}$ & $\overline{0}$ \\
\hline 0.650 & 0.409 & $t$ & 6 \\
\hline--- & --- & $\mathbf{u}$ & 6 \\
\hline-- & --- & $r$ & 8 \\
\hline
\end{tabular}

Angenommene Oerter 1877.0.

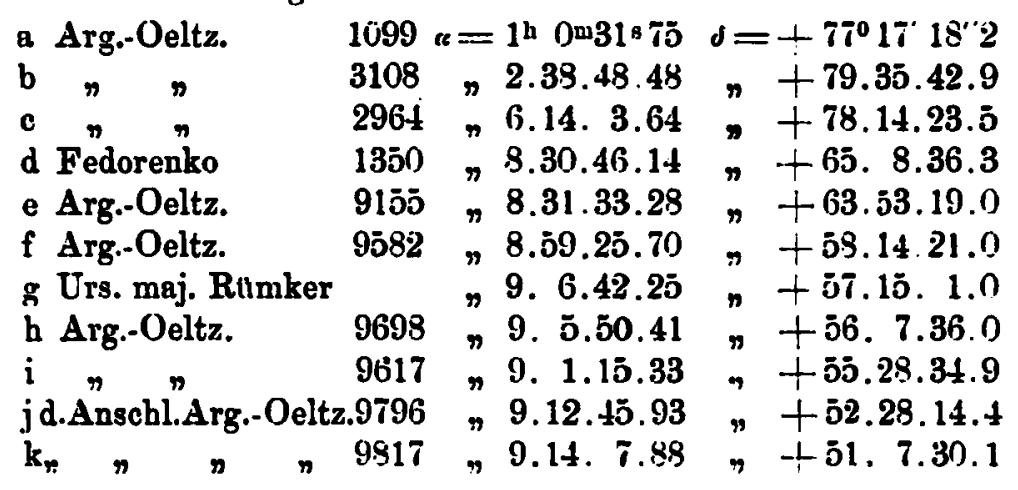




\begin{tabular}{|c|c|c|}
\hline 1 Arg.-Oeltz. & $=9^{\mathrm{h}} 14^{\mathrm{m}} 8^{\mathrm{s}} 44$ & $\delta=+50^{\circ} 4^{\prime} \quad 1^{\prime \prime} 1$ \\
\hline 1990 & $\eta 9.20 \cdot 1.34$ & $\eta+49.24 . \overline{3} 1$ \\
\hline n Arg.-Oeltz. & $\eta 9.16 .5 \overline{7} .01$ & $"+47.51 .33 .2$ \\
\hline o d. Anschl. B. & $\eta 9.9 .4 .91$ & $n+47.19 .44 .2$ \\
\hline p Arg.-Oeltz. & $\eta 9.19 .44 .82$ & $n+46.17 .7 .7$ \\
\hline 10002 & $" 9.25 .58 .37$ & +45.50 .19 \\
\hline r d. Anschl. Arg. & $\Rightarrow 9.19 .41 .64$ & +45.27 .49 .2 \\
\hline s B. W. 672. & $" 9.33 .4 .81$ & +44.32 .53 .8 \\
\hline t d. Anschl. B.W. 513 u. 14 &, 9.26 .17 .32 & +43.51 .56 .3 \\
\hline u Lal. 18977 u. 18978 & $\eta 9.34 .2321$ & $+40.19 \cdot 9.4$ \\
\hline r B. W. 918,19 น. 20. & $\eta 9.44 . \tilde{0} 3.62$ & +36.52 .49 .9 \\
\hline w Argel. 6243 & $\eta 5.42 .46 .44$ & $+57.5 \overline{5} .50 .4$ \\
\hline$x \quad r \quad 6686$ & $" 6.9 .750$ & +56.18 .3 .5 \\
\hline y Merid.-Best. & $n \quad 6.28 .1989$ & $n+54.20 .11 .0$ \\
\hline$z$ Argel, 7612 & $n 7.2 .54 .46$ & +49.25 .254 \\
\hline
\end{tabular}

Kremsmünster 12. Sept. $187 \%$.

G. Strasser.

\section{Elemente und Ephemericle}

des ron J. Coggia in Marseille am 13. September entdeckten Cometen, berechnet von Dr. J. Holetscheck.

Circular der kaiserlichen Akademie der Wissenschaften in Wien.

Bis zum Schlusse der Rechnungen waren die folgenden Beobachtungen bekannt geworden:

Ort $187 i$ mitl. Ortsz. app. a $\mathbb{L}^{4}$

1. Marseille Sept. 13

2. " $" 14$

3. Pola"

4. Mailand

5. Leipzig

b. Pola

7. Leipzig

$\therefore$ Wien

9.

10. Strassburg $16^{\mathrm{h}} 4\left(\mathrm{j}_{\mathrm{m}}\right.$ s $5 \mathrm{~s} 33^{\mathrm{m}}$ ह

$14.38 .8 \quad 8.32 \quad 3 \quad 10$

14.25 .18

13.40 .5

8.31 .24 .64

$8.30 .4 \overline{5} .56$

12.32. 4

४ 31$) .3 .46$

8.29 .56 .84

829.14 .99

829.12 .81

8.29 .10 .34

5. 29.12 .32 app. $d \&$

$+45^{\circ} 30^{\circ}$

48.14. 0.6

47.58 .16 .3

$47.4 \mathrm{i} .50 . \mathrm{I}$

47.26 .7 .6

4723.27 .2

47. 8.23 .8

47. 7.37 .6

47. 7. 3.2

$+47.6 .54 .5$
Beobachter

Coggia

J. Palisa

Schiaparelli

Peter

J. Palisa

Boruhus

Weiss

A. Palisa

Winnecke

Aus den Positionen 2, 4 und dem Mittel aus 8 und 9 ergab sich das folgende Elementensystem: $\mathrm{T}=187 \mathrm{i}$ September $27.93 \overline{3} 3$ mittl. Berl. Zeit.

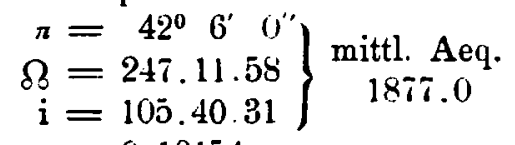

$\log \mathrm{q}=0.181 \mathrm{~b} 4$

Darstellung der mittleren Beobachtung (B.-R.):

$$
\begin{aligned}
\mathrm{d} \lambda \cos \beta & =+14^{\prime \prime} \\
\mathrm{d}_{\beta} & =-4 .
\end{aligned}
$$

